\title{
INGLATERRA DEJÓ DE SER UNA ISLA: INFLUENCIA EUROPEA SOBRE EL PROCESO CIVIL INGLÉS*
}

NeIL ANDREWS**

\section{INTRODUCCIÓN}

En este artículo consideraremos las conexiones entre el proceso civil inglés y Europa, ambos al nivel de principios fundamentales concernientes a tópicos específicos.

Indudablemente, el mayor impacto del régimen europeo de derechos humanos ha sido la decisión de crear una Corte Suprema del Reino Unido para asegurar la completa separación de las funciones entre la legislatura y el sistema judicial, asegurando que el Lord Chancellor (Lord Canciller) ${ }^{1}$-esto es, el Ministro de Justicia- ya no pueda ocupar el puesto de juez.

En lo que resta de este artículo vamos a examinar otras dos conexiones entre el proceso civil inglés y la justicia civil europea: las restricciones de la Unión Europea (UE) sobre las sanciones para el cumplimiento del disclosure (revelación) ${ }^{2}$ de información durante una medida cautelar conservativa (freezingorder) ${ }^{3}$, y la Directiva Europea sobre Mediación (2008).

\footnotetext{
*Traducción de Álvaro Pérez Ragone (Profesor de Derecho Procesal Civil de la Pontificia Universidad Católica de Valparaíso - traducción que forma parte del proyecto de investigación Fondecyt № 1111021 Principios de la tutela ejecutiva del crédito) y Pablo Bravo Hurtado (Universidad Católica de Temuco). Correo electrónico: alvaro.perez@ucv.cl. Colaboración recibida el 16 de julio y aprobada el 20 de septiembre de 2011.

** M.A., B.C.L. (Oxon.), Profesor de Derecho Procesal Civil nacional, internacional y comparado, Derecho Civil, catedrático de la Facultad de Derecho , Universidad de Cambridge (Clare College).

${ }^{1}$ El Lord High Chancellor of Great Britain es un cargo político inglés que no tiene un símil suficientemente cercano entre nosotros. Es el segundo más importante después del Primer Ministro inglés (es decir, como un vicepresidente), pero con competencias en la administración de los tribunales como un Ministro de Justicia, que es Juez de la House of Lords y participa en la legislatura (N. del T.).

2 El Disclosure está definido como el "acto o proceso de hacer conocido algo que era previamente desconocido; una revelación de hechos". Black's Law Dictionary, 2a Edición, p. 207 (N. del T.).

${ }^{3}$ Una "freezingorder" cumple en el derecho inglés una función similar a las medidas cautelares. Mientras éstas se dirigen a bienes específicos, la freezingorder tiene por destinatario al requerido respecto de todos sus bienes.
} 


\section{El SURGIMIENTO DE LOS PRINCIPIOS}

Los principios de la justicia civil se han vuelto un campo importante del estudio comparado 4 . En particular, los principios procesales constitucionales han adquirido preeminencia ${ }^{5}$. La discusión académica internacional es próspera sobre los principios fundamentales (así notablemente los escritos de Hazard, ${ }^{6}$ Jolowicz, ${ }^{7}$ Kawano, ${ }^{8}$

${ }^{4}$ La bibliografíacomparatista en lengua inglesa incluye: Jolowicz, J.A., On Civil Procedure, Cambridge University Press, 2000 (y la que se cita a seguir cronológicamente): Cappelletti, M.; Perillo, J., Civil Procedure in Italy, La Haya, 1995; CAPpellettı, M. (editor), "Civil Procedure", enInternational Encyclopaedia of Comparative Law, La Haya, y Tubinga, volumen XVI, 1976; LanGBeIN, J., 'The German Advantage in Civil Procedure' 52 Univ. of Chi. LR, 1985, pp. 823-866; DAMASKA, M., The Faces of Justice and State Authority: A Comparative Approach to the Legal Process, New Haven, 1986; Cappellettı M., The Judicial Process in Comparative Perspective, Oxford University Press, Oxford,1989; StORme, M. (editor), Approximation of Judiciary Law in the EU, Dordrecht, 1994; ZUCKERMAN, AAS (editor), Civil Justice in Crisis: Comparative Perspectives of Civil Procedure, Oxford University Press, Oxford, 1999; ReChBerger, W.; Klicka, H. (editores), Procedural Law on the Threshold of a New Millenium, XI World Congress of Procedural Law, Center for Legal Competence, Viena, 2002; Asser, D et al., `A summary of the interim report on Fundamental Review of the Dutch Law of Civil Procedure', 2003, p. 8 ZZ PInt pp. 329-387; Storme, M. (editor), Procedural Laws in Europe - Towards Harmonization, Maklu, Amberes/ Apeldoorn, 2003; Storme, M.; Hess, B. (editores), Discretionary Power of the Judge: Limits and Control, Kluwer, Dordrecht, 2003; MurRay, Peter; Stürner, Rolf, German Civil Justice, Durham, 2004; Van RheE, CH. (editor), The Law's Delays: Essays on Undue Delay in Civil Litigation, Amberes y Oxford, 2007; Van Rhee, CH., European Traditions in Civil Procedure, Intersentia and Hart, Oxford, 2005; Trocker, $\mathrm{N}^{\circ}$; VARAno, V. (editores), The Reforms of Civil Procedure in Comparative Perspective, Turin, 2005; CHASE, Oscar, Hershroff, Helen, Silberman, Linda Varano, Vincenzo, Taniguchi, Yasuhei, Zuckerman, Adrian, Civil Procedure in Comparative Context, Thomson West, 2007; Pellegrini Grinover, A.; Calmon, R. (editores), Direito Processual Comparado: XIII World Congress of Procedural Law, Editora Forense, Río de Janeiro 2007, pp. 201-242; Uzelac, A.; Van Rhee, CH. (editores), Public and Private Justice, Amberes y Oxford, 2007; Deguchi, M. and Storme, M. (editores), The Reception and Transmission of Civil Procedural Law in the Global Society, Maklu, Amberes, 2008. And on 'transnational principles', Storme, M. (editor), Approximation of Judiciary Law in the European Union, Gantes 1994 and ALI/UNIDROIT's Principles of Transnational Civil Procedure, Cambridge University Press, 2006; on this project, KRONKE, H. (editor), special issue of the Uniform Law Review, 2002, Volumen VI; Andenas, M., ANDRews, N., NazzINI, R. (editores), The Future of Transnational Commercial Litigation: English Responses to the ALI/UNIDROIT Draft Principles and Rules of Transnational Civil Procedure, British Institute of Comparative and International Law, Londres, 2006; STÜRner, Rolf, 'The Principles of Transnational Civil Procedure', Rabels Zeitschrift, 2005, pp. 201-254.

${ }^{5}$ Así, Goldstein, Stephen, 'The Influences of Constitutional Principles on Civil Procedure in Israel', 17 Israel LR, 1982, pp. 467-510; and "Constitutional Norms of Civil Procedure as Reflected in the ALI/ UNIDROIT Principles of Transnational Civil Procedure" en Estudios en Homenaje a Hector Fix-Zamudio; The Proposed ALI/UNIDROIT Principles and Rules: the Utility of Such A Harmonization Project VI Uniform LR, 2001-4, pp. 789-801.

${ }^{6}$ ALI/UNIDROIT's Principles of Transnational Civil Procedure, Cambridge, Cambridge University Press, 2006.

${ }^{7}$ Jolowicz , J.A., On Civil Procedure, cit. nota n. 4.

${ }^{8}$ MASANORI KaWANo (Editor) A New Framework for Transnational Business Litigation', Mohr Siebeck, Tubinga, 2010; Stürner, Rolf and KAwANo, Masanori (editores), Current Topics of International Litigation, Mohr Siebeck, Tubinga, 2009; national studies: Andrews Neil, English Civil Justice and Remedies: Progress and Challenges: Nagoya Lectures, Shinzan Sha Publishers, Tokyo, 2007; Ervo, Laura (editora), Civil Jus- 
Murray, ${ }^{9}$ Shetreet, ${ }^{10}$ Storme, ${ }^{11}$ Stürner, ${ }^{12}$ y Taruffo $)^{13}$ en Europa. Ello, en parte se debe a que la Convención de Estados Europeos, incluido el Reino Unido, deben cumplir con la jurisprudencia de la Corte de Estrasburgo ${ }^{14}$ concerniente a las garantías contenidas en el artículo 6(1) de la Convención Europea de Derechos Humanos. Otra fuente importante de los mayores principios procesales son los Principios del Procedimiento Civil Transnacional de la UNIDROIT/American LawInstitute, publicado en $2006^{15}$, aunque no son vinculantes.

\section{Artículo 6(1), Convención Europea de Derechos Humanos}

La Ley de Derechos Humanos de 1998 (Humans Rights Act), que entró en vigencia en octubre de 2000, hace que la Convención Europea de Derechos Humanos sea directamente aplicable a y por los tribunales ingleses. El artículo 6(1) de la Convención establece ${ }^{16}$ : "Derecho a un Juicio Justo (Fair Trial): en la determinación de los derechos civiles y obligaciones o cualquier imputación criminal en su contra, todos tienen derecho a una audiencia justa y pública (fair and publichearing) dentro de un tiempo razonable y por un tribunal independiente e imparcial establecido por la ley".

Laimportante sistematización del principio fundamental comprende los siguientes elementos:

tice in Finland, Jigakusha Publishing, Tokyo, 2009; Eslugues-Mota, Carlos; Barona-Vilar, Silvia (editores), Civil Justice in Spain, Jigakusha Publishing, Tokyo, 2009; KenGyel, Miklos; HarsaGı,Viktoria, Civil Justice in Hungary, Jigakusha Publishing, Tokyo, 2010; AnDRews, Neil, Contracts and English Dispute Resolution, Jigakusha Publishing, Tokyo, 2010; MANIOTIS, Dimitris; TsantinIs, Spyros, Civil Justice in Greece, Jigakusha Publishing, Tokyo, 2010; SCHмIDT, Stephanie, Civil Justice in France, Jigakusha Publishing, Tokyo, 2010; DE Cristofaro, Marco; Trocker, Nicolo (editores), Civil Justice in Italy, Jigakusha Publishing, Tokyo, 2010.

${ }^{9}$ Murray, Peter; Stürner, Rolf, German Civil Justice, Durham, USA, 2004.

${ }^{10}$ Publicaciones en Mount Scopus International Standards of Judicial Independence.

11 Storme, M. (editor), Approximation of Judiciary Law in the European Union, Gante,1994; ver igualmente Storme, M. (editor), Procedural Laws in Europe - Towards Harmonization, Maklu, Amberes/ Apeldoorn 2003; Storme, M.; Hess, B. (editores), Discretionary Power of the Judge: Limits and Control Kluwer, Dordrecht, 2003.

${ }^{12}$ Murray, Peter; Stürner, Rolf, German Civil Justice, Durham, USA, 2004; ALI/UNIDROIT's Principles of Transnational Civil Procedure, Cambridge, Cambridge University Press, 2006.

${ }^{13}$ ALI/UNIDROIT's Principles cit. nota n. 6.

${ }^{14}$ La Corte Europea de Derechos Humanos tiene su asiento en la capital de la región francesa de Alsacia, la ciudad de Estrasburgo. De ahí que se refiere a ella en breve como "Corte de Estrasburgo" (N. del T.).

15 ALI/UNIDROIT's Principles cit. nota n. 6.

16 Human Rights Act 1998, art. 1(3), que incorpora la Convención Europea de Derechos Humanos en el derecho del Reino Unido; Grocz, S.; Beatson, J.; Duffy, P., Human Rights: The 1998 Act and the European Convention, segunda edición, 2008; Janies, M.W.; Kay, R.S.; Bradley, A., European Human Rights Law: Text and Materials, tercera edición, Oxford UniversityPress, Oxford, 2008; CLAYTON, R.; TOMLINSON, H., The Law of Human Rights, segunda edición, Oxford University Press, Oxford, 2008. 
(a) el derecho a una "audiencia justa" (fairhearing): este es un concepto amplio y abarcador ${ }^{17}$ : (i) el derecho a estar presente en una audiencia con contradictorio; (ii) el derecho a la igualdad de armas; (iii) el derecho a una oportunidad justa para ofrecer prueba (fair presentation of evidence); (iv) el derecho a interrogar y contrainterrogar (cross examination) al testigo de la contraparte; (v) el derecho a una sentencia fundamentada ${ }^{18}$;

(b) "una audiencia pública": incluyendo el derecho a un pronunciamiento público de la sentencia; ${ }^{19}$

(c) "un juicio dentro de un tiempo razonable", y

(d) "una audiencia ante un tribunal independiente ${ }^{20} \mathrm{e}$ imparcial ${ }^{21}$ establecido en la ley".

\section{Derogación de la House of Lords y la creación de la Corte Suprema del Reino Unido}

Indudablemente, el impacto más significativo de la Convención Europea de Derechos Humanos fue la decisión de derogar el tradicional rol judicial del Lord Chancellor y reconstituir el Comité de Apelación de la House of Lords (Cámara de los Lores) en la Corte Suprema del Reino Unido. La Corte Suprema se establece el 1 de octubre de 2009. Los eventos se sucedieron del siguiente modo.

El 12 julio del año 2003, el gobierno laborista, liderado por el entonces primer ministro "Tony" Blair, anunció su plan de derogar la ancestral función judicial del Lord Chancellor, quien degradaba la función judicial de la House of Lords, y transferir esa función a la nueva Corte Suprema. En 2005, la ley de reforma constitucional ${ }^{22}$ fue promulgada, llevando a la derogación de la House... en 2009. El 28 de julio de 2009, los Magistrados (Lords) dictaron su última sentencia de una apelación ordinaria en la Cámara de House of Lords

\footnotetext{
${ }^{17}$ Clayton y Tomlinson, cit. nota n. 16.

${ }^{18}$ Andrews, Neil, English Civil Procedure, Oxford, Oxford University Press, 2003 parágrafos 5.39 a 5.68 .

${ }^{19}$ Ibíd., para 4.59 hasta el final del capítulo; conforme a lo mencionado por las autoridades de Estrasburgo en los parágrafos 7.21 a 7.79 .

${ }^{20}$ Starrs contra Ruxton 2000 JC 208, 243; 17 de noviembre 1999, The Times (High Court of Justiciary) por Lord Reed; Millar contra Dickson [2002] 1 WLR 1615, PC; ANDrews, Neil, English Civil cit. nota n.18, parágrafos 4.02 a 4.27 (independencia judicial).

${ }^{21}$ Porter contra Magill [2002] 2 AC 357, HL.

22 "Reform! Reform! Don't talk to me about reform. Aren't things bad enough already?" adjudicado a Astbury J., como hizo notar Neuberger, D., 'The Supreme Court: Is the House of Lords "Losing Part of It self'" (2 de diciembre 2009), p. 13.
} 
(el último caso respecto de la cuestión del suicidio asistido ${ }^{23}$. El 1 de octubre de 2009, la Corte Suprema del Reino Unido sesionó por primera vez.

La principal razón para la derogación del Comité de apelación de la House of Lords fue la necesidad percibida para así desmantelar la triple responsabilidad del Lord Chancellor, el así llamado "doble eje" (universal joint) de la Constitución británica ${ }^{24}$ : en primer lugar, su estatus combinado del Ministro (dentro de la responsabilidad ministerial del Poder Ejecutivo de gobierno, para el Departamento de Estado, conocido como la oficina del Lord Chancellor, ahora ministro de justicia); en segundo lugar él era también presidente de la House of Lords, y de este modo tenía competencia legislativa; y tercero, para finalizar, era juez, capaz de presidir las apelaciones conocidas por la House of Lords o en Privy Councipr.

La Corte Europea de Derechos Humanos enMcGonnel v. UK (2000), en un caso sobre disposiciones legales en la isla de Guernsey, un "mini-sistema jurídico" dentro de las islas británicas, ha señalado la necesidad de que haya una completa separación entre la función judicial, ejecutiva y legislativa. En el caso McGonnell la Corte Europea de Derechos Humanos sostuvo que el Reino Unido ha infringido este requisito porque el Alguacil (Bailiff) de Guernsey (un juez y que integrara la Legislatura de Guernsey) intervino en casos civiles que involucran proyectos de ley que han sido promulgados cuando le tocó a él presidir la cámara legislativa de la isla ${ }^{26}$. La Corte de Estrasburgo sostuvo que esa confusión del rol legislativo y judicial "es incompatible con el requisito de independencia e imparcialidad" que exige el artículo 6 (1) de la Convención Europea de Derechos Humanos. Esta Corte sostuvo igualmente que "cualquier participación directa en la tramitación de una ley o en los reglamentos del ejecutivo es probable que sea suficiente para poner en duda la imparcialidad judicial de una persona que está llamada, acto seguido, a dirimir una disputa sobre qué razones existen para permitir un cambio en la redacción de la ley o

\footnotetext{
${ }^{23}$ Neuberger, D, The Supreme Court, cit. nota n. 22, p. 2.

${ }^{24}$ Le Sueur, A., 'From Appellate Committee to Supreme Court: A Narrative', en Blom-CoOper, L., Dickson, B., Drewry, G. (editores), pp. 66-70. Sobre los roles del Law Lord, R. Cornes, 'McGonnel contra UK, the Lord Chancellor and the Law Lords' (2000) PL 166, 174; WoODHOUSE, D., The office of Lord Chancellor: time to abandon the judicial role-the rest will follow, 2002, p. 22 LS 128, 141 y ss.

${ }^{25}$ Consejo consultor del Estado, que se conforma por la necesidad en la monarquía de un órgano consultor del Estado.

26 (2000) 30 EHRR 289, ECHR; on which, Le Sueur, A., 'Access to Justice Rights in the United Kingdom', 2000, 5 EHRLR 457, 467; CORNES, R, 'McGonnell contra UK, the Lord Chancellor and the Law Lords', 2000, p.166; Woodhouse, D, 'The Office of Lord Chancellor', 1998, p. 617 y del mismo autor 'The Office of Lord Chancellor: Time to Abandon the Judicial Role...', 2002, p. 22 LS 128, 141-3; para una visión general WoOdHousE, D., The Office of the Lord Chancellor, Hart, Oxford, 2001.
} 
reglamentos de que se trata" ${ }^{27}$. La misma Corte sostuvo que cuando una persona ha presidido una cámara legislativa, ella debe quedar inhabilitada para juzgar cualquier caso civil o criminal que requiera la interpretación de la disposición promulgada ${ }^{28}$.

La decisión del caso McGonnell despertó dudas sobre la competencia del Lord Chancellor para intervenir en casos judiciales. Ello, porque él simultáneamente encabeza el poder judicial, es un miembro del gabinete de gobierno a cargo del Departamento de Estado (Lord Chancellor'sDepartment), y además es integrante de la cámara legislativa de la House of Lords $^{29}$.

De hecho, últimamente los Lord Chancellors no han sesionado judicialmente en muchas ocasiones por estar desarrollando otras funciones en las que se ocupan. Incluso cuando integran la Corte, debe ser un miembro elegido entre los cinco que componen los comités de apelaciones de la House of Lords ${ }^{30}$. La investigación de Lord Bingham reveló que desde 1945 hasta el 2003 las sesiones para las apelaciones han sido de un promedio de ocho días al año, y que la decimoquinta parte del período Law Lord (magistrado permanente) era de 120 días al año en la corte ${ }^{31}$. Por un corto tiempo, al comienzo del caso McGonnel/(2000), ${ }^{32}$ se logró un acuerdo con el Lord Chancellor ${ }^{33} \mathrm{com}-$ prometiéndose a que no sesionaría en ningún caso criminal (en que el Estado necesariamente está involucrado en la persecución del imputado), y solamente sesionaría en casos civiles en donde el gobierno no tenga ningún interés en el resultado del juicio, ya sea directa o indirectamente ${ }^{34}$. De este modo la ley

\footnotetext{
${ }^{27}$ McGonnell case, 2000, 30 EHRR 289, ECHR, p. 55.

${ }^{28}$ Ibíd., p. 57.

${ }^{29}$ Para ver el listado de la pluralidad de roles en el derecho inglés de los Lords ver Cornes, R., 'McGonnell contra UK, cit. nota n. 26, 166, 174; para un análisis adicional ver WOODHOUSE, D., 'The office of Lord Chancellor: time to abandon the judicial role-the rest will follow', 2002, p. 22 LS 128, 141 y ss.

${ }^{30}$ Lord HalLSHAm enuncia que durante sus dos períodos en la House of Lords appeal estuvo un total de 81 días en su oficina; y Lord MACKAY 60 días: HL Deb 17 Febrero 1999, vol 597, col 738; BLOM-CoOPER, L., Dickson, B., DrewryG (editores), The Judicial House of Lords: 1876-2009, Oxford University Press, Oxford, 2009, p. 232.

${ }^{31}$ BINGHAM, T, 'The Old Order Changeth' 2006, p.122 LQR 211, 217.

32 (2000) 30 EHRR 289, ECHR.

${ }_{33}$ Para un análisis del debate parlamentario sobre la separación de poderes, HL Deb 17 February 1999, volumen 597, 710-39; el former Lords Chancellors HalLSHAM sosteniendo, que ello no implicaba un problema 'The Office of Lord Chancellor and the Separation of Powers', 1989, p. 8 CJQ 308; Lord MACKAY, 'The Lord Chancellor in the 1990s', 199 1, p. 44 CLP 241; crítico Woodhouse, D. 'The Office of Lord Chancellor',1998, PL 617; OLIVER, D. 'The Lord Chancellor, the Judicial Committee of the Privy Council and Devolution',1999, PL 1; Munro, C., Studies in Constitutional Law, segunda edición, 1999, pp. 314-317, 328, 331.

34 Lord IRVINE LC in HL Deb 17 de febrero 1999, volumen 597, col. 736 y HL Deb 2 March 2000 vol. 610, col. 657; Dickson, B. y Carmichael, P. (editores), The House of Lords: Its Parliamentary and Judicial Roles, 1998.
} 
de reforma constitucional del año $2005^{35}$ ha despojado de su rol judicial a la antigua oficina del Lord Chancellor. Ha dejado de ser un juez. A cambio, es simplemente un representante del Ejecutivo, un Ministro de la Corona, y un miembro del gabinete. En efecto, la ley de 2005 estableció que el Lord Chancellor no necesita siquiera tener formación jurídica.

El cambio también fue defendido por los constitucionalistas más puristas ${ }^{36}$ que deseaban separar la House of Lords judicial de la House of Lords legislativa y por tanto (i) los Law Lords estarían físicamente separados de la legislatura y (ii) ya no se les permitiría participar en los debates legislativos ${ }^{37}$. Una vez que la decisión fue tomada, el Lord Chancellor fue desde entonces reducido a un ministro "no-judicial" (ver abajo) y simultáneamente el terreno quedó libre para la creación de la Corte Suprema. Como Lord Bingham explicósi: "Con el Lord Chancellor que deja de tener un puesto judicial, y su centro de gravedad profesional moviéndose hacia las sedes departamentales..., ya había poco... que justificara la continua presencia de los Law Lords en la legislatura a la que han asistido en el último tiempo, por cierto a contribuir relativamente poco". Esto fue una "victoria total" para los constitucionalistas puristas que la postularon.

La nueva Corte Suprema del Reino Unido ahora está expresamente aislada de cualquier 'contaminación legislativa o ejecutiva': ninguno de sus jueces está involucrado en el proceso legislativo, o participan en el Gobierno. No sería más que llorar sobre la 'leche constitucional' derramada preguntarse sobre si exisitió o no alguna necesidad de alterar las tradiciones asentadas de la House of Lords. Pero aún es un asunto debatido si acaso fue necesario crear la Corte Suprema y aniquilar el rol judicial de la House of Lords.

\footnotetext{
${ }^{35}$ Ya anticipando la Constitutional Reform Act 2005, Lord STEYN (ahora retirado como Lord of Appeal), ya en el año 2002 en su 'AllSoulsCollege, NeillLecture', había criticado el multifacético rol del Lord Chancellor: SteYn, 'The Case for a Supreme Court', 2002, p. 118 LQR 382.

36 Lord IRVINe LC in HL Deb 17 febrero 1999, vol 597, col 736 and HL Deb 2 marzo 2000 vol. 610, col. 657; Dickson, B. and Carmichael, P. (editores) The House of Lords: Its Parliamentary and Judicial Roles, 1998.

37 Los Law Lords no jubilados habían ya comenzado a evitar la participación en los debates parlamentarios sobre los asuntos políticos del partido, y algunos incluso habían prometido no dirigirse ni hablar en forma individual por ante el órgano legislativo, ver los detalles en BLOM-COOPER, L., DiCKSON, B. Drewry, G. (editores), The Judicial, cit., nota n. 30, e ibíd., Hope, David, capítulo 11, especialmente pp. 175 y ss.

${ }^{38}$ Bingham, T., The Old Order Changeth, 2006, LQR 211, pp. 122 221-2.
} 


\title{
V. TUtelA CAUTELAR CONSERVATIVA (FREEZING RELIEF) ${ }^{39}$ : VÍNCULOS TRANSNACIONALES DENTRO Y FUERA DE EUROPA
}

\begin{abstract}
La Regulación Europea sobre Competencia (European Jurisdiction Regulation) exige que los tribunales de los Estados Miembros pongan a disposición su sistema de tutela de derechos para apoyar los litigios que se llevan en los tribunales de los otros Estados Miembros ${ }^{40}$. De hecho, la High Court inglesa puede conceder una medida cautelar conservativa (freezing injuction) en apoyo de un proceso civil pendiente o eventual ${ }^{41}$ en todo el mundo, sea que la respectiva jurisdicción
\end{abstract}

\begin{abstract}
39 El trabajo de investigación por excelencia es el de GEE, S., Commercial Injunctions, Londres, 2004; ver igualmente ANDrews, Neil, English Civil cit. nota n. 18, capítulo 17; Zuckermanon Civil Procedure, Londres, 2006, parag. 9.139 y ss.; GoldREIN, I.S. (editor), Commercial Litigation: Pre-emptive Remedies, actualizado al 2010; para consultar una extensa bibliografía al respecto sobre este tema ver ANDREWs, op. cit., 17.01 n. 7 y ANDREws, Neil 'Provisional and Protective Measures: Towards a Uniform Provisional Order', Uniform L. Rev. (Revdrunif) volumen VI, 2001, pp. 931-949 (interesante artículo que contiene el análisis de un anteproyecto pensando en un código internacional o practices relacionadas con este tipo de medidas cautelares, conservación de pruebas y órdenes de declaración de bienes o activos). Lo relevante de ello es que no se reduce a la jurisdicción inglesa o del Commonwealth: REDFERN, A., in Newman, L.W. and HiLL, R.D. (editores), The Leading Arbitrators' Guide to International Arbitration, Berna, 2004, p. 217 y ss. y Knoepfler. F. 'Les Mésures Provisoires et l'Arbitrage' en Cadiet, L., JeUland, E., ClaY, T. (editores), Médiation et Arbitrage: Alternative Dispute Resolution-Alternative a la justiceo ujustice alternative? Perspectives comparatives, LexisNexis Litec, Paris, 2005; MustiLL, M. y Bord, S., Commercial Arbitration, Londres, 2001, volúmenes adicionales, pp. 314-6, 323-4; la High Court tiene competencia según lo dispuesto en el número 44, 1996 Act, para ordenar a petición de parte y sin previa audiencia del requerido una cautelar conservatoria o de búsqueda de bienes o aporte de información relevante como medidas anexas y para resguardar un proceso arbitral, siempre que la materia sea urgente (Mobil Cerro Negro Ltd. contra Petroleos de Venezuela SA[2008] EWHC 532 (Comm), comentado por JoHnson, Adam, 2008, CJQ 433-444; ello ya que el tribunal arbitral por sí carece de la competencia para ordenar este tipo de medidas a petición de una de las partes; VAN HOUTTE, H, 'Ten ReasonsAgainst a Proposalfor Ex Parte InterimMeasures of Protection in Arbitration', 2004, Arbitration International 85, p. 20; BAY$\mathrm{KITCH}, \mathrm{A}$. and TRUONG, J., 'Innovations in International Commercial Arbitration: Interim Measures a Way Forward or Back totheFuture', 2005, The Arbitrator and Mediator 95, p. 25; TROCKER, N, 'Provisional Remedies in Transational Litigation: the Issue of Jurisdiction: a Comparative Outline', 2009, Int'I Lis 48-56; ANDREws, Neil, 'Provisional and Protective Measures: TowardsanUniform Provisional Order', Uniform L Rev (Revdrunif), volumen VI, 2001, p. 931; GoldsteIN, Stephen, 'RevisitingPreliminaryRelief in Light of the ALI/UNIDROIT Principles and the New Israeli Rules' en Studia in honorem: Pelayia Yessiou-Faltsi, Atenas, 2007, pp. 273-296. Más aún, los principios de litigación civil "transnacional" del American Law Institute/UNIDROIT reconocen la importancia de dichas órdenes y protección cautelar: American Law Institute/UNIDROIT's Principles of Transnational Civil Procedure, Cambridge University Press, 2006, principle 8, y sobre ello Goldstein, Stephen, ibíd.
\end{abstract}

${ }^{40}$ Dicey, Morris and Collins, Conflict of Laws (14 Edi., London, 2006), 8-024; G. MAHER - B.J. ROdGer, 'Provisional and Protective Remedies: The British Experience of the Brussels Convention', 1999, ICLQ 302, p. 48.

41 ... proceedings have been or are to be commenced in a [Brussels or Lugano] Contracting State [or a Member State]') según 25(2), 1982 Act y ver la siguiente nota. In C-104/03, St Paul Dairy Industries NV contra UnibelExser BVBA [2005] ECR I-3481 el Tribunal de Justicia Europeo sostuvo que la definición contenida en el artículo 31 (antiguo artículo 24 de la Convención de Bruselas de 1968) del Reglamento de Competencia sobre cautelares o medidas provisionales o no incluye un procedimiento preliminar 
extranjera sea parte o no del régimen de competencias de Bruselas y Lugano ${ }^{42}$. La medida cautelar conservativa inglesa, formalmente denominada "Mareva Injuction" $^{\prime 43}$ es una orden in personam que exige al demandado abstenerse de disponer de sus bienes, usualmente activos en cuentas bancarias. La medida cautelar conservativa no le otorga al demandante ningún tipo de interés sobre la propiedad de los activos del demandado ${ }^{44}$.

Las cautelares conservativas inglesas (freezingin juctions) operan en un primer momento inaudita pars a sola petición del demandante (ex parte), usualmente antes que el procedimiento principal haya comenzado. Una vez que esta medida ex parte se ha concedido, el mérito de ella es revisado en una audiencia inter partes. Existe un formulario estándar para esas solicitudes ${ }^{45}$. A los jueces se les exige hacer un uso restringido de estas medidas. En general, sólo un juez de la High Court ${ }^{46}$ puede conceder estas medidas.

El demandado incurrirá en desacato (contempt of court) si infringe la medida cautelar $^{47}$, y puede ser multado, arrestado o el secuestro (sequestration) de sus bienes. Una vez notificado el banco, o incluso un tercero, puede arriesgar penas por desacato ${ }^{48}$ (por ejemplo, multas pagaderas al tribunal) si "a sabiendas" obs-

para la obtención de pruebas con el fin de determinar si un caso puede o no prosperar en las palabras del Tribunal de Justicia: "a measure ordering the hearing of a witness for the purpose of enabling the applicant to decide whether to bring a case, determine whether it would be well founded and assess the relevance of evidence which might be adduced in that regard is not covered by the notion of provisional, including protective, measures".

${ }^{42}$ Civil Jurisdiction and Judgments Act 1982 ver 25; Civil Jurisdiction and Judgments Act 1982 (Interim Relief) Order 1997 (SI 1997, № 302) (1982 Act, 25) que se refiere al deber del Reino Unido para dar efectividad al artículo 24 de la Convención de Bruselas de 1968 (actualmente artículo 31 del Reglamento Jurisdicción № 44/2001); también la regulación del 1 de abril de 1997, jurisdicción de High Court para ordenar medidas provisionales en virtud de la Ley de 1982, que excluyó del Convenio a los Estados no miembros, y que se aplica a los procedimientos civiles fuera del ámbito restrictivo establecido en el artículo 1 del Convenio de Bruselas y su sucesor, el Reglamento de Competencia: (CE) № 44/2001 de 22 de diciembre de 2000, sobre competencia judicial, el reconocimiento y la ejecución de las sentencias en materia civil y asuntos comerciales [2001] OJ L 12/1.

${ }^{43}$ Las Civil Procedure Rules 25.1(1) y s. refiere nuevamente a estas órdenes provisorias (injunction), disposición por cierto que había sido ratificada en el numeral 37(3), de la Senior Courts Act 1981.

${ }^{44}$ Cretanor Maritime Co Ltd contra Irish Marine Maritime Ltd [1978] 3 All ER 164, CA; Capital Cameras Ltd contra Harold Lines Ltd [1991] 1 WLR 54; Flightline contra Edwards [2003] 1 WLR 1200, CA.

${ }^{45}$ Practice Direction (PD) (25).

${ }^{46}$ PD (25): Los Masters como jueces de distrito pueden ordenar estas medidas sólo en casos excepcionales.

${ }^{47}$ Motorola Credit Corpn contra Uzan (N 2) [2003] EWCA Civ 752; [2004] 1 WLR 113, CA [45]-[58], [148]-[156]; Federal Bank of the Middle East Ltd contra Hadkinson [2000] 2 All ER 395, 411, CA.

${ }_{48}$ Anexo a la PD (25); Z Ltd contra A [1982] QB 558, 567, CA, comentado por Eveleigh, LJ; conf. con el caso Supply of Ready Mixed Concrete ( $\left.N^{\circ} 2\right)$ [1995] 1 AC 456, HL; también con los autos Att-Gen contra Newspaper Publishing plc [1997] 3 All ER 159, 169, CA. 
taculiza el funcionamiento de la medida cautelar ${ }^{49}$, si continúa las instrucciones del cliente o gira sus cheques, excepto cuando dichos actos están permitidos por la medida cautelar ${ }^{50}$.

Una medida cautelar conservativa casi siempre se complementa con una orden de declaración de bienes (asset disclousure order), exigiendo del demandado que revele los detalles de sus activos en Inglaterra (o, cuando corresponda, en otros lugares $)^{51}$. Además, un tercero puede ser obligado a proveer esa información si ha colaborado, incluso inocentemente, con la mala fe habiendo mala fe de otro o de la parte requerida ${ }^{52}$.

Las medidas cautelares conservativas transfronterizas son ahora comunes ${ }^{53}$. Trevor Hartley (2010) ha resumido el impacto que han tenido estas medidas sobre los bienes, información o personas fuera de Inglaterra y Gales ${ }^{54}$. El demandante puede obtener tanto una medida in personam que afecta los bienes relevantes en el extranjero y una orden de declaración de bienes (disclosure) que obligue al demandado a proveer la información relacionada con ellos relacionadas. La orden de manifestación de bienes transfronteriza tiene una mayor importancia práctica y táctica que la medida cautelar conservativa principal ${ }^{55}$. Esto es porque lo que sigue es meramente una "operación de retención" para darle al deman-

\footnotetext{
49 Anexo a la PD (25).

50 Z Ltd contra A [1982] QB 558, CA.

51 Derby \& Co Ltd contra Weldon ( $\mathrm{N}^{\circ} 1$ ) [1990] Ch 48, CA; Derby \& Co Ltd contra Weldon ( ${ }^{\circ}$ s. 3 \& 4) [1990] Ch 65, 86, 94-5, CA; Bank of Crete SA contra Koskotas [1991] 2 Lloyd'sRep 587, CA; LA Collins (1989) 105 LQR 262, 286 y ss.; Comp. McLachlan, 'The Jurisdictional Limits of Disclosure Orders: Transnational Fraud Litigation' (1998) ICLQ , p. 3.

${ }^{52}$ Sobre la regla de los meros testigos ver Norwich Pharmacal Co contra Customs and Excise [1974] AC 133, HL and, in the present context, Bankers Trust Co contra Shapira [1980] 1 WLR 1274, CA, Arab Monetary Fund contra Hashim (N 5) [1992] 2 All ER 911, HoffmAnn, J.; ANDrews, Neil, English Civil, cit. nota n.18, parágrafos 26.102 a 26.128 .
}

53 Anexo a las PD (25); Babanaft Co SA contra Bassatne [1990] Ch 13, CA; Republic of Haiti contra Duvalier [1990] QB 202, CA; Derby \& Co contra Weldon ( $N^{\circ}$ 1) [1990] Ch 48, CA; Derby \& Co Ltd contra Weldon (Nº 3 \& 4) [1990] Ch 65, CA; ColuINs, LA, 'The Territorial Reach of Mareva Injunctions', 1989, LQR 262-99, p.105; ColuINS, L.A., capítulos VIII y IX, en Essays in International Litigation, Oxford, Oxford University Press, 1993; DICEY- MORRIS-Coluins, Conflict of Laws, 14 edición, Londres, 2006, pp. 8-11 y ss.; los tribunales ingleses no pueden ordenar medidas de aseguramiento para la ejecución con posterioridad al fallo de acuerdo al Art 47(1), Jurisdiction Regulation No 44/2001, Banco Nacional de Comercio Exterior SNC contra Empresa De Telecomunicationes de Cuba SA [2007] EWCA Civ 662 (dejado sin efecto en Steel J año [2006] EWHC 19 (Comm)); noted L Merrett [2007] CLJ 495-8.

${ }^{54}$ HARTLEY, T 'Jurisdiction in Conflict of Laws: Disclosure, Third-Party Debt and Freezing Orders', 2010, LQR 194, pp. 126 201-205 (sobre el disclosure), 210-221 (sobrelasfreezing injunctions).

${ }^{55}$ DiceY- Morris-Colıins, cit. nota n.53, pp. 8-011 y ss.; Crédit Suisse Fides Trust SA v Cuoghi [1998] QB 818, 827-8, CA, comentado por MiLlett LJ. 
dante el tiempo necesario para solicitar al tribunal(es) extranjero respectivo la apropiada tutela principal complementaria o sustantiva ${ }^{56}$.

A los tribunales ingleses usualmente se lo solicita que otorguen tutela conservativa para apoyar grandes acciones civiles por fraude (sean procesos llevados en Inglaterra o en otra parte), usualmente con pluralidad de codemandados. Algunos de ellos no tendrán su domicilio en Inglaterra. Según la Regulación de la Jurisdicción en la Unión Europea, existe la facultad para agregar un nuevo codemandado a los procedimientos civiles, incluso si esa persona no tiene domicilio en Inglaterra ni tampoco bienes allí ubicables e identificable. Por cierto, los tribunales ingleses tienen jurisdicción bajo el mismo régimen respecto al primer demandado ${ }^{57}$. Los codemandados que se suman de este modo pueden no tener bienes en Inglaterra. Parte del objetivo de procurar una tutela conservativa será auxiliarse de la orden de manifestación de bienes para ubicar y tasar los activos de los codemandados. ¿Pero qué podría hacer un tribunal inglés para que se cumpla con la orden de declarar los bienes en contra de un codemandado con residencia en el extranjero y que no tiene bienes disponibles para ser embargados en Inglaterra, y que ha incumplido dicha orden emitida en Inglaterra? Después de todo, a menos que tontamente ponga un pie en suelo inglés, él no puede ser encarcelado ni multado. Fuera de la jurisdicción, y sin bienes que embargar dentro de Inglaterra, él es de facto inmune contra los procedimientos de desacato (contempt of court). Impávidos frente a esta dificultad práctica, los tribunales ingleses han empleado potentes "órdenes subsidiarias" (unless order) y sanciones de "sentencia en rebeldía" (ver más abajo) en contra de los demandados ausentes.

Esta cuestión se basa en un caso complicado que en 2007 fue llevado a la Corte Europea de Justicia. En este caso el codemandado Gambazzi, tenía su residencia en Suiza. Él fue agregado como codemandado a un proceso judicial civil inglés en 1996. La demanda incluía acusaciones de fraude en una escala astronómica. El tribunal inglés ordenó a Gambazzi que hiciera una manifestación completa de sus bienes a nivel mundial. Esta orden de manifestación de bienes fue una ramificación de la medida cautelar conservativa principal en contra de

\footnotetext{
${ }^{56}$ Babanaft International Co SA contra Bassatne [1990] Ch 13, 41, CA, comentado por Nicholls L.J.

${ }^{57}$ Council Regulation (EC) No 44/2001 del 22 de diciembre del 2000 la competencia, el reconocimiento y ejecución de resoluciones judiciales en materia civil y mercantil; el Art. 6 establece: `A person domiciled in a Member State may also be sued: (1) where he is one of a number of defendants, in the courts for the place where any one of them is domiciled, provided the claims are so closely connected that it is expedient to hear and determine them together to avoid the risk of irreconcilable judgments resulting from separate proceedings...'. Para estos efectos, la House of Lords sostuvo en 1 AC 1 [2002] que el demandado ya lo es desde el momento mismo en que se admite la demanda contra él, siempre que tenga su domicilio en Inglaterra en ese momento no afectando sus cambios de domicilio (en los hechos por los que huyen del país) antes que se le notifique de la demanda al codemandado no domiciliado en Inglaterra, no pudiendo oponerse fundados en el Art. $6 \mathrm{CEDH}$.
} 
Gambazzi. Para añadir fuerza a dicha orden, el tribunal inglés en 1998-9 emitió una serie de órdenes "supletorias", "prohibitivas", en ausencia o rebeldía. ${ }^{58}$

La Corte de Apelaciones en el caso Marcan Shipping (London) Ltd. contra Kefelas (2007) explicó cómo funcionan estas "órdenes supletorias" 59 . El efecto procurado era que, si Gambazzi no cumplía completa y oportunamente con la orden de manifestación respectiva, la orden de la Corte se haría efectiva en el sentido que: (i) se desestimará la defensa en el fondo de Gambazzi; (ii) se le excluirá de representación legal para participar en los procesos civiles que determinan su responsabilidad y cuantifiquen el monto, y (iii) se le permitirá a los demandantes obtener una sentencia condenatoria ejecutable por los montos demandados que determine el tribunal. Después Gambazzi no cumplió completamente con la "orden supletoria", ya que omitió hacer una manifestación completa de sus bienes, y el demandante utilizó a su favor el elemento (iii) (ver arriba) para obtener un sentencia en rebeldía. Esto fue por las cantidades finalmente determinadas por el tribunal inglés sin la participación de Gambazzi. Esa enorme sentencia en rebeldía fue confirmada en la apelación por la Corte de Apelaciones inglesa en $2004^{60}$.

La siguiente fase relevante de este litigio fue el intento del acreedor de obtener reconocimiento y ejecución de la sentencia ejecutoriada inglesa en Italia en contra de los bienes de Gambazzi en Italia (si los hubiere). Pero los tribunales italianos en 2007 remitieron a la Corte Europea de Justicia la cuestión acerca de si, sobre estos hechos, hay una cuestión de orden púbico acerca de la legitimidad del procedimiento que sirviera de objeción para los tribunales de un Estado miembro. Esa objeción les permitiría, si lo consideran necesario, rechazar el reconocimiento y la ejecución de esta sentencia inglesa pronunciada en rebeldía. El argumento de Gambazzi fue que la sentencia inglesa se había pronunciado sin haberle dado oportunidad de contradecir el resultado, o el monto de lo fallado en su contra.

Así, cuando se hizo la remisión a la Corte Europea de Justicia, la Corte d'Appelo di Milano (27 de junio de 2007) señaló que el artículo 27(1) de la Convención de Bruselas (1968) (la Convención es aplicable en el tiempo a los

\footnotetext{
${ }^{58}$ ARDEN L.J. resume la historia procesal de este caso en [2004] EWCA Civ 827, pp. 3 y ss.

${ }^{99}$ [2007] EWCA Civ 463; [2007] 1 WLR 1864, ver pp. 33-36 para una lúcida síntesis de los principios, véase también las pp. 14, 28 y 30, el tribunal consideró que, de acuerdo a la CPR 3.1 (3) el tribunal puede emitir al menos una orden un condicional o o sujeta a caducidad, en complemento con la CPR 3.8 (1) (las sanciones en con aplicación inmediata) y CPR 3.9 (atenuación de las sanciones), y CPR 3.5 ( que consagra el derecho de la parte demandada para iniciar un proceso con costas cuando se rechazan las defensas o no se acoge la demanda de la contraparte o en caso de rebeldía), el tribunal señaló ya precedentes antes y después de las CPR, , en este caso ver ZUCKERMAN, AAS, 'HowSeriouslyShouldUnlessOrders be Taken?', 2008, CJQ , pp. 1-7 , 27 (ver para ello la nota siguiente).
}

${ }^{60}$ [2004] EWCA Civ 827. 
procedimientos respectivos anteriores) ${ }^{61}$ permite que la corte requerida rechace reconocer y ejecutar una sentencia civil pronunciadapor los tribunales de un Estado miembro, si la sentencia se ha obtenido de un modo que sea "contrario al orden público en el Estado cuyo reconocimiento se solicita". Con todo, esto no permite al tribunal de ejecución rechazar un acto simplemente sobre la base de una diferencia entre su derecho sustantivo o principios de su propio sistema jurídico y aquellos que se aplicaron en el tribunal de origen. En su lugar, se establece que debe haber un vicio procesal grave en el otorgamiento de la sentencia respectiva por el tribunal de origen.

En Marco Gambazzi v. Daimler-Chrysler Candalnc and CIBC Mellon Trust Company (Caso C-394/07) de 2 de abril de 2009, la Corte Europea de Justicia sostuvo:

"El tribunal de un Estado en el cual es solicitada la ejecución, debe tomar en cuenta, en atención a la cláusula de orden público a la que refiere el artículo 27(1) de la Convención de Bruselas (1968), el hecho que el tribunal del Estado de origen falló sobre la demanda del actor sin escuchar al demandado que compareció ante ella, pero que fue excluido del procedimiento por no cumplir la obligación impuesta por una orden hecha anteriormente en el mismo procedimiento. Esto, si tras una evaluación comprehensiva de los procedimientos y a la luz de las circunstancias, resulta que esa medida de exclusión constituye una infracción manifiesta y desproporcionada al derecho del demandado a ser escuchado".

En 2011, los tribunales italianos aplicando este pronunciamiento recién citado, sostuvieron que en el caso Gambazzi no se podía lograr impugnar la sentencia inglesa en rebeldía ${ }^{62}$.

Desde la década de los 90, cuando se pronunciaron las sentencias en rebeldía en el caso Gambazzi, la Corte de Apelaciones Inglesa en el caso Motorola (2003) reconoció que los tribunales no deben ser draconianos en la implementación de las órdenes de declaración de bienes en contra de codemandados con residencia extranjera. Incluso si ellos son culpables del

\footnotetext{
${ }^{61}$ Sobre este punto ver la sentencia de la Corte d'Appello de Milán del 27 de junio del 2007, que sostiene en la p. 3 que: "Es cierto que de conformidad con el artículo 66 del Reglamento 44/2001 del Consejo ['sobre competencia, reconocimiento y ejecución de resoluciones judiciales en materia civil y mercantil '], dado que las órdenes del tribunal ingles son de 1998 y del 17 de diciembre 1999, los procedimientos de ejecución se rigen por el Convenio de Bruselas. Sin embargo, dado que las disposiciones pertinentes de ambos instrumentos son idénticas, la referencia errónea a las normas aplicables no da lugar a la nulidad del decreto dado que las condiciones de ejecución de conformidad con los artículos 25 y siguientes han sido correctamente aplicadas. Por lo tanto, de conformidad con el principio de que los actos procesales deben siempre que sea posible mantener su efecto, incluso si son extrínsecamente defectuosos, la irregularidad no da lugar a la nulidad de la resolución que los aplica" (cf. Cass. no. 365/2003).

${ }^{62}$ Agradezco al Profesor Marco de Cristofaro de Padova por esta información.
} 
incumplimiento, ya que en todo caso se les permitió participar en la apelación contra las órdenes de manifestación de activosy en la tutela conservativa principal ${ }^{63}$.

Más aún, la Corte de Apelaciones inglesa en Motorola Credit Corpn contra Uzan (2004) se trató el problema de una eventual parte que no es residente dentro de Inglaterra y que tampoco tiene bienes allí64. Se dijo que sería relevante considerar: "si en un caso en que se espera que haya resistencia y desobediencia a una orden jurisdiccional, el tribunal estará emitiendo una orden que no pueda ser ejecutada". En el caso Motorola, este factor justificó revocar la tutela conservativa destinada a dos codemandados con residencia en Turquía, que no tenían bienes en Inglaterra ${ }^{65}$. Sin embargo, porque los otros codemandados sí tenían residencia y propiedades en Inglaterra, el tribunal mantuvo la tutela conservativa en contra de éstos ${ }^{66}$.

Finalmente, es notable que el tribunal inglés formó tres regímenes diseñados para proteger a los demandados y terceros de la injusticia que pudieran padecer fuera de la jurisdicción inglesa.

El primero de estos tres regímenes (según lo prescrito en Dadourian Group International Inc. contra Simms, 2006), está diseñado para proteger al demandado en contra de una ejecución opresiva de una medida cautelar inglesa transfronteriza en una jurisdicción extranjera ${ }^{67}$. En segundo lugar, los terceros

63 [2003] EWCA Civ 752, p. 55] la Corte de Apelaciones sostuvo que : "...al mismo tiempo es totalmente obsoleto que la actitud desafiante de los demandados, como se desprende de las pruebas de que, se parte de una serie de retrasos tácticos y lamentable falta de franqueza en dos jurisdicciones, se tiene en cuenta que las apelaciones de los acusados son esencialmente defensivas dada la naturaleza. Su postura en esta jurisdicción ha sido uno de resistencia a una serie de órdenes restrictivas e intrusivas solicitada por el demandante en un procedimiento extranjero, en lugar de la invocación de un voluntario de las facultades de la Corte para su propio beneficio. Esto nos parece influir en la proporcionalidad de impedir que, como partes en el desprecio, de lo que sería su derecho a apelar contra las órdenes de congelación a la que las órdenes de interrogatorio eran auxiliares. En todas las circunstancias, tenemos la opinión de que los acusados deben ser escuchadas y sus argumentos tratados como dirigidos a todos los de sus recursos y solicitudes que nos ocupan".

${ }_{64}$ [2004] 1 WLR 113, CA, p. 115; sobre el temprano case law ANDREws, Neil, English Civil cit. nota n.18, párrafo 17.74 y ss.; ver igualmente JoHnson, Adam in ANDENAS, Mads-AnDrews, Neil-NAZZINI, Renato (editores), The Future of Transnational Civil Litigation, reimpreso por el British Institute of International and Comparative Law, 2006, capítulo 11.

${ }^{65}$ [2004] 1 WLR 113, CA, pp. 125 y 126.

66 ibid, pp. 127 y 128.

67 [2006] EWCA Civ 399; [2006] 1 WLR 2499; [2006] CP Rep 31, p. 25 con comentarios sobre cada uno de los casos en p. 26 y ss.; comentado igualmente por RutheRFORD, T. (2006) NLJ 837 en los puntos 1, 4 y 5 (citado infra) son especialmenteimportantes los considerandos que establecen: "permission should be just and convenient... and...not oppressive to the parties to the English proceedings or to third parties who may be joined to the foreign proceedings" (...)"permission should not normally be given in terms that would enable the applicant to obtain relief in the foreign proceedings which is superior to the relief given by the orders [órdenes o medidas inglesas]" (...) "evidence in support of the application for permis- 
que residan en otras jurisdicciones podrán ser sancionados por los tribunales ingleses si no cumplen una medida cautelar conservativa inglesa sólo dentro de los límites establecidos de la llamada "disposición Babanaft" ${ }^{68}$. El resultado de esto es que un tercero, como el banco del demandado, será sancionable en Inglaterra sólo si tuviere en la práctica el control de la sucursal en el extranjero ya por incumplir con la tutela conservativa inglesa, ya por continuar cumpliendo las órdenes financieras del demandado respectivo, y constituya allí un acto de incumplimiento o vulneración por el demandado de la medida cautelar conservativa inglesa.

$\mathrm{Y}$ en tercer lugar, en el caso de conflicto entre la medida conservativa inglesa y el Derecho extranjero, la "disposición Báltico" permite al demandado respectivo o a un tercero cumplir mediante el respectivo contradictorio del Derecho Extranjero ${ }^{69}$.

\section{LA DIRECTIVA EUROPEA DE MEDIACIÓN (2008)}

El Ministro de Justicia de Inglaterra y Gales (2010) reportó70 "una duplicación de las mediaciones desde el 2007". En un sentido general y vago, la directiva Europea de mediación recepta bien esta tendencia global. Pero uno ya casi no necesita este instrumento para subrayar la realidad moderna de la resolución de conflictos.

sion should contain all the information... necessary to make the judge to reach an informed decision, including evidence as to the applicable law and practice in the foreign court, evidence as to the nature of the proposed proceedings to be commenced and evidence as to the assets believed to be located in the jurisdiction of the foreign court and the names of the parties by whom such assets are held".

${ }^{68}$ Ello incorporado en el anexo a las PD (25), 19; esta protección se origina en Babanaft Co SA contra Bassatne [1990] Capítulo 13, CA: "The terms of this Order do not affect or concern anyone outside the jurisdiction of this Court until it is declared enforceable or is enforced by a Court in the relevant country and then it shall affect him only to the extent it has been declared enforceable or has been enforced UNLESS such person is: a person to whom this Order is addressed or an officer of or an agent appointed by a power of attorney of such a person; or a person who is subject to the jurisdiction of this Court and (a) has been given written notice of this Order at his residence or place of business within the jurisdiction of this Court and (b) is able to prevent acts or omissions outside the jurisdiction of this Court which constitute or assist in the breach of the terms of this order".

${ }^{69}$ [2002] 1 All ER 717, CA; se debe mencionar que el origen de esta prevision está en el caso Baltic Shipping contra Translink Shipping Ltd [1995] 1 Lloyd's Rep 673, CLARKE, J; la disposición está ahora contenida en el Anexo a las PD (25), 20: "...nothing in this order shall, in respect of assets located outside England or Wales, prevent [en este caso el banco que no es parte] or any of its subsidiaries from complying with what it reasonably believes to be its obligations, contractual or otherwise, under the laws and obligations of the country or state in which those assets are situated or under the proper law of any bank account in question; and any orders of the courts of that country or state provided reasonable notice of any application for such an order by [en este caso el banco que no es parte] or any of its subsidiaries(to the extent such notice is permitted by the criminal law of such country or state), is given to the claimant's solicitors".

70 Ministerio de Justicia, 'Implementation...Paper', Londres, 2010, (paper de trabajo) p. 10. 
La Directiva Europea de Mediación (2008) ${ }^{71}$ ha tenido un impacto sustantivo pequeño, porque está limitada a las disputas transfronterizas. Así, el artículo $2^{\circ}$ establece que la Directiva se ocupa sólo de la mediación en materias 'civiles y comerciales', esto es, cuando las partes tienen su domicilio o residencia habitual en distintos Estados miembros, o el Estado miembro anfitrión es distinto al Estado miembro de las partes. Esta es una categoría pequeña. El Ministerio de Justicia Inglés (2010) estimó que en los años 2007, 2008 y 2009, hubo menos de 50 mediaciones dentro de Inglaterra con una parte de otro Estado miembro ${ }^{72}$.

La Directiva concretamente trata acerca de cuatro materias: (1) control de calidad; (2) ejecución rápida; (3) confidencialidad, y (4) prescripción. Además de exhortar a los europeos a considerar la mediación, uno se pregunta por qué se introdujo la Directiva misma. Quizás es una mera tendencia centralista de inmiscuirse en algo nuevo. No se justifica una discusión detallada acerca de (1), (2) y (4). Ellas son simples materias de detalle. En cuanto a (3) es atractivo el énfasis de la Directiva sobre la confidencialidad, pero nuevamente es muy poco el aporte en detalles. El artículo $7^{\circ}$ no desarrolla la distinción insinuada en su texto, entre la confidencialidad en la información generada dentro de la mediación, y la competencia y obligación de los mediadores como testigos en un proceso judicial. En Inglaterra hay una red compleja de reglas y doctrinas concernientes al 'secreto de la mediación' ${ }^{\prime 73}$.

Con todo, la Directiva al menos Ilama la atención sobre el fenómeno difundido de la mediación. Sólo incidentalmente subraya la naturaleza 'voluntaria' de la mediación. Esta importante reafirmación del principio fundamental se realiza en el Preámbulo de la Directiva:

(13) La Mediación ofrecida en esta Directiva debe ser un procedimiento voluntario en el sentido que las partes están ellas mismas a cargo de ese proceso, pueden organizarlo como deseen y terminarlo en cualquier momento. Sin embargo, debe ser posible bajo el Derecho nacional, que los Tribunales establezcan plazo para el proceso de mediación. Es más, los tribunales deben poder llamar la atención a las partes sobre la posibilidad de la mediación cada vez que sea apropiado.

(14) Nada de lo dispuesto en esta Directiva debe ir en perjuicio de la legislación nacional que haga uso de la mediación obligatoria o la sujeta a incenti-

71 Directiva Europea 2008/52/EC del Parlamento Europeo, luego del Consejo Europeo (del 21 mayo del 2008) sobre ciertos aspectos de la mediación en materias civil y comercial, en Diario Oficial L 136, 24/05/2008 P. 0003 - 0008.

72 Ministerio de Justicia, 'Implementation...Paper', Londres, 2010, (paper de trabajo), p. 12.

${ }^{73}$ Ver RAmsey, J. comentario sobre el caso Farm Assist Limited (in liquidation) v. The Secretary of State for the Environment, Food and Rural Affairs ( $N^{\circ}$ 2) [2009] EWHC 1102 (TCC); [2009] B.L.R. 399; 125 Con. L.R. 154, especialmente pp. 44, 45 y ss. 
vos o sanciones, siempre que esa legislación no impida a las partes ejercer sus derechos de acceso al sistema judicial...'.

Por lo tanto, los autores de la Directiva han reconocido en el párrafo (13) que la mediación no puede ser impuesta sobre las partes. Pero los mismos autores también reconocen en el párrafo (14) que los incentivos estatales o sanciones dispuestas por los tribunales civiles, puedan ser usados para fomentar el uso de la mediación. Los incentivos no son problemáticos, las sanciones sí son materia de controversia. Ellas deben ser administradas cautelosamente. Una sanción debe ser aplicada sólo en apoyo a un deber limitado: este deber está restringido a considerar la mediación, cuando la posibilidad sea sugerida por la otra parte ${ }^{74}$ o por el tribunal. ${ }^{75}$

\section{CONCLUSIONES 76}

Por razones de espacio, esta exposición no contiene una discusión detallada sobre los diversos reglamentos de la Unión Europea que han sido receptados e incorporados obligatoriamente dentro de los Estados miembros bajo la autoridad del marco legal de la UE. Además del Reglamento sobre las Competencias $(2001)^{77}$, y las regulaciones adicionales del proceso civil comunitario como: los Reglamentos sobre Notificaciones ${ }^{78}$, Prueba ${ }^{79}$, Título Ejecutivo Europeo ${ }^{80}$,

\footnotetext{
${ }^{74}$ Un ejemplo reciente es el caso Rolf contra De Guerin [2011] EWCA Civ 78.

${ }^{75}$ Dunnett v. Railtrackplc [2002] 1 WLR 2434, CA, p. 13 y ss.

${ }^{76}$ Storskrubb, E., Civil Procedure and EU Law: A Policy Area Uncovered, Oxford UP, 2008; Crifo, C., "Civil Procedure in the European Order: an Overview of the Latest Development", en DwYER, D. (editor), The Civil Procedure Rules Ten YearsOn ,Oxford UP, 2009, capítulo 19.

77 Regulación del Consejo Europeo (EC) 44/2001 del 22 diciembre del 2001 sobre reconocimiento y ejecución de sentencias en materias civil y comercial.

${ }_{78}$ Regulación del Consejo Europeo (EC) 1393/2007 del 13 noviembre del 2007 sobre notificación de instrumentos judiciales y extrajudiales dentro de los Estados Miembros en material civil y comercial, con lo que se deroga la regulación (EC) 1348/2000 de 29 mayo 2000 sobre la materia; STORSKRUBB, E., Civil Procedure and EU Law: A Policy Area Uncovered, Oxford UP, 2008, capítulo 6.

79 Regulación del Consejo Europeo (EC) 1206/2001 of 28 May 2001 en cooperación judicial entre los tribunals de los Estados Miembros para la rendición de prueba en material civil y comercial; STORSRRUBB, Civil Procedure, cit. nota n. 78, capítulo 7.

${ }^{80}$ Regulación del Consejo Europeo (EC) 805/2004 of 21 April 2004 por la que se constituye un título ejecutivo judicial europeo para demandas no controvertidas; StORSKRUBв, E., Civil Procedure, cit. nota n.78, capítulo 9.
} 
Orden de Pago (o monitorio) Europea ${ }^{81}$, y el Procedimiento Europeo de Pequeñas Causas ${ }^{82}$.

Los principales puntos que han sido de contribución al derecho inglés pueden resumirse en los siguientes:

1. El Derecho inglés está ahora sujeto directamente a la Convención Europea de Derechos Humanos en virtud de la Ley inglesa de Derechos Humanos de 1998. Una consecuencia de ello, espectacular y controversial de esta nueva cultura de los derechos humanos, ha sido la decisión de eliminar la oficina judicial del Lord Chancellor que existía desde hace mucho tiempo y su reemplazo por el Comité de Apelación de la House of Lords dentro de la Corte Suprema del Reino Unido. Ello, recuérdese, es resultado dramático de la decisión de Estrasburgo concerniente a las disposiciones de la isla de Guernsey, en McGonnel $v \cup K(2000)$.

2. En temas más específicos, en este artículo consideramos otros dos ejemplos de la interacción del proceso civil inglés con el Derecho Europeo (tanto el Derecho de la Unión Europea como la jurisprudencia de Estrasburgo sobre Derechos Humanos).

3. En el contexto de la tutela cautelar conservativa (freezingrelief), los tribunales ingleses y la legislación europea (artículo 24 de la Convención de Bruselas de 1968, ahora el artículo 31 del Reglamento sobre Competencia) en conjunto han logrado efectivamente un régimen armonioso y expansivo para inmovilizar bienes y para obtener información acerca de ellos. Pero la decisión de la Corte Europea de Justicia en el caso Gambazzi (2009) ha trazado una línea a las sanciones inglesas frente al incumplimiento de las órdenes de declaración de esa información. En ese caso, los tribunales italianos estaban instruidos acerca de qué orden público procesal podía justificar la negativa a reconocer una sentencia en rebeldía inglesa basada en la negativa de Gambazzi de manifestar toda su información patrimonial relevante. Sin embargo, la secuela de esto se manifiesta en que los tribunales italianos (2011) han sostenido que la sentencia en rebeldía inglesa debe ser reconocida y ejecutada.

4. La Directiva Europea de Mediación (2008) fue considerada en Inglaterra como una incursión irrelevante de las autoridades europeas dentro de una esfera dinámica y expansiva de la justicia civil, donde la práctica inglesa ya se había

81 Regulación del Consejo Europeo (EC) 1896/2006 of 12 December 2006 dando origen a la orden de pago o monitorio europeo implementado en las CPR Parte 78, sección I (desde el 12 de diciembre del 2008); Storskrubb, E, Civil Procedure, cit. nota n. 78, capítulo 12; Crifo, C., "Civil Procedure", cit. nota n. 76, capítulo 19, pp. 370-374.

82 Regulación del Consejo Europeo (EC) 861/2007 of 11 July 2007 estableciendo el procedimiento europeo para menor cuantía receptado en las CPR Parte 78, sección II (desde 1 de enero del 2009); Storskrubb, E., "Civil Procedure" cit. nota n. 78, capítulo 13; Crifo C., "Civil Procedure", cit. nota n. 76, pp. 374-378. 
expandido. La importancia real de la Directiva más bien pareciera ser simbólica, antes que concreta y real. La Directiva sólo señala la necesidad para los Estados Miembros de tomarse en serio la mediación. Al parecer la importancia de la mediación de algunos Estados Miembros no era autoevidente (como se reportan los colegas italianos y alemanes). 
